\title{
Charcot Spine following chronic spinal cord injury: an analysis of 201 published cases
}

\author{
Ryan Solinsky $\mathbb{1 D}^{1,2} \cdot$ Jayne M. Donovan ${ }^{3,4} \cdot$ Steven C. Kirshblum ${ }^{3,4}$
}

Received: 3 August 2018 / Revised: 16 October 2018 / Accepted: 18 October 2018 / Published online: 9 November 2018

(c) International Spinal Cord Society 2018

\begin{abstract}
Study design Analysis of published cases

Objectives To identify and describe symptoms, radiologic findings, treatment strategies, and reoccurrence of Charcot spine in individuals with spinal cord injury (SCI).

Methods This analysis included all English articles published prior to October 2017, describing Charcot spine after SCI as identified by multiple reviewers. Articles were excluded if Charcot spine was attributed to alternative conditions. Individual level data were available for $94 \%$ of reported cases. Outcomes included demographic factors, injury characteristics, clinical presentation, radiologic findings, management, and reoccurrence.

Results Fifty included papers described 201 individuals with SCI who developed Charcot spine. $86 \%$ of individuals had paraplegia and $93 \%$ of individuals had a neurologically complete injury. Mean length of initial spinal fusion spanned 7.7 vertebral bodies $(\mathrm{SD}=3.9)$. The most common presenting symptoms were back pain $(56 \%)$, spinal deformity $(48 \%)$, and crepitus (34\%). Vertebral body destruction (83\%), osteophytes $(61 \%)$, and endplate destruction $(57 \%)$ were commonly reported on radiographs. Reoccurrence of Charcot spine was described in 19\% of cases after initial treatment.

Conclusion Charcot spine after SCI commonly presents with low back pain and radiologic evidence of vertebral body destruction. Cases have been described more often in individuals with paraplegia and neurologically complete injuries. Surgical management is often pursued. A high rate of reoccurrence of Charcot spine in individuals with SCI after initial treatment has been reported.
\end{abstract}

\section{Introduction}

Charcot spine, a degenerative spinal arthropathy, has classically been associated with the tabes dorsalis complication of tertiary syphilis. The etiology for this condition has been postulated to be due to loss of protective sensory neuronal

Electronic supplementary material The online version of this article (https://doi.org/10.1038/s41393-018-0216-6) contains supplementary material, which is available to authorized users.

Ryan Solinsky

rsolinsky@mgh.harvard.edu

Spaulding Rehabilitation Hospital, Boston, MA, USA

2 Department of Physical Medicine \& Rehabilitation, Harvard University, Boston, MA, USA

3 Kessler Institute for Rehabilitation, West Orange, NJ, USA

4 Department of Physical Medicine \& Rehabilitation, Rutgers New Jersey Medical School, Newark, NJ, USA innervation leading to repetitive microtrauma and inflammation that degrades the intervertebral joint [1]. However, this classic etiologic mechanism has been partially revised in recent years [2], specifying that either nociceptive sensory and/or proprioceptive deficits may contribute to Charcot spine.

As advanced cases of syphilis are far less common than in the past, Charcot spine has more recently been reported in individuals with other conditions, including spinal cord injury (SCI). Similar to tabes dorsalis, previous literature [3, 4] notes that repetitive microtrauma may be a contributing issue in this population, with increased stretch of neighboring ligaments not eliciting compensatory, protective muscle contractions in individuals with SCI. It has been proposed that this increased, uncompensated spinal motion may be further multiplied when paired with the long spinal fusions ( $>5$ levels) [4], increasing intervertebral joint forces in already vulnerable adjacent segments. Other potential contributing factors include changes in bone homeostasis below the level of injury and loading forces associated with 
daily activities after SCI such as transfers. This combination of factors following SCI may contribute to a unique pathophysiologic mechanism for patients who develop Charcot spine versus previous reports of this condition as a complication of tabes dorsalis or peripheral neuropathy.

Clinically, Charcot spine in individuals with SCI can be a significant complication, progressing to the point of pain, deformity, spinal instability, and even neurologic loss of function, before being diagnosed [5]. Nevertheless, it remains a relatively rare condition following SCI, with an estimated prevalence of one out of every 220 patients [6]. As such, despite being proposed in multiple texts, many of the assumptions of presentations and recurrence remain speculative. This is evident and further amplified by the large number of case studies and small case series on the topic, with very few aggregate studies to extrapolate broad trends and enhance collective knowledge. The single large review of cases of Charcot spine, is limited by a heterogeneous population of patients with SCI, congenital pain insensitivity, and tabes dorsalis [7]. Given the potential altered biomechanics and unique conditions accompanying motor/sensory/autonomic paralysis, our knowledge on Charcot spine is incomplete and may be insufficient for addressing care specific to individuals with SCI.

The objective of this study was to review available published cases of Charcot spine following SCI in an effort to strengthen the collective understanding of patient presentations and management of this condition.

\section{Methods}

Published English literature was searched with Google Scholar for the terms "Charcot Spine", "Charcot arthropathy spine", "Charcot spinal arthropathy", "neuroarthropathy spine", "spinal neuroarthropathy", and "neuropathic spine" with no defined start date and ending in October 2017. Google Scholar was used to achieve a more inclusive, potentially non-indexed search. Abstracts were reviewed and cases were excluded if they were not in English or were found to be due to congenital pain insensitivity, infection, syphilis, Friedreich's ataxia, GuillainBarre syndrome, chronic inflammatory demyelinating polyneuropathy, or other peripheral neuropathies (diabetes, leprosy, alcoholic, etc.). Given the potential for further confounding from pre-morbid altered bone metabolism (Andersson lesions/psuedoarthroses), cases where individuals were diagnosed with ankylosing spondylitis prior to their SCI were also excluded. Finally, as many early cases of SCI were treated with laminectomy without fusion, fractures through a laminectomy site (without fusion) were excluded, given altered loading through this potentially weakened vertebral column. Full texts of each article with potential reported cases for inclusion were compiled and the references of each were searched to find any further applicable manuscripts detailing cases on Charcot spine in individuals with SCI.

Included manuscripts were reviewed by two authors and key metrics were extracted including: patient age, gender, American Spinal Injury Association Injury Impairment Scale (AIS) grade, neurological level of injury (NLI), chronicity of SCI at time of Charcot spine diagnosis, initial fusion location and length, clinical presentation, delay between symptom onset and diagnosis, radiologic findings, levels of Charcot spine, management, recurrence of Charcot spine, and years of follow up monitoring. We defined recurrence as subsequent Charcot spine occurring in the same individual at a second, unique location within the spinal column following their primary treatment. This differed from nonunion, where evidence for ongoing Charcot spine may appear at the previously effected site after treatment. No prior review protocol existed. Both traumatic and non-traumatic sources of SCI were included, though classified accordingly. Efforts were taken to extract individual data for each patient, though aggregated results alone were reported in some case series. Senior authors of case series with aggregate data were approached for individual data when email contact information was listed.

Of note, there was a wide range of dates that the reports originated from and the definition of "complete" SCI had correspondingly changed over the course of the study. This was a detail that was unable to be controlled for with available information. As such, studies that mentioned "complete injury" were assumed to mean AIS A [8]. Studies that mentioned only "incomplete injury" were not included in subanalyses involving AIS, due to imprecision of this description. Cases where a specific NLI was not reported were excluded for analyses involving this metric.

Descriptive statistics were calculated for demographic data, presentations, and management details. Heteroscodastic t-tests were utilized for analyses of variables, with base $P$ values of $<0.05$ treated as significant following appropriate Bonferroni correction $(p<0.003)$. Following analysis, data were archived and made available for future research utilization/expansion as ongoing cases are reported at Harvard Dataverse (https://doi.org/10.7910/DVN/ RUTHC4).

\section{Results}

Eighty papers were found that met the initial search parameters and appeared to contain potential case report information of Charcot spine following SCI (Supplemental Table 1). Following application of our exclusion criteria (Fig. 1), 50 manuscripts of case reports/series reporting 


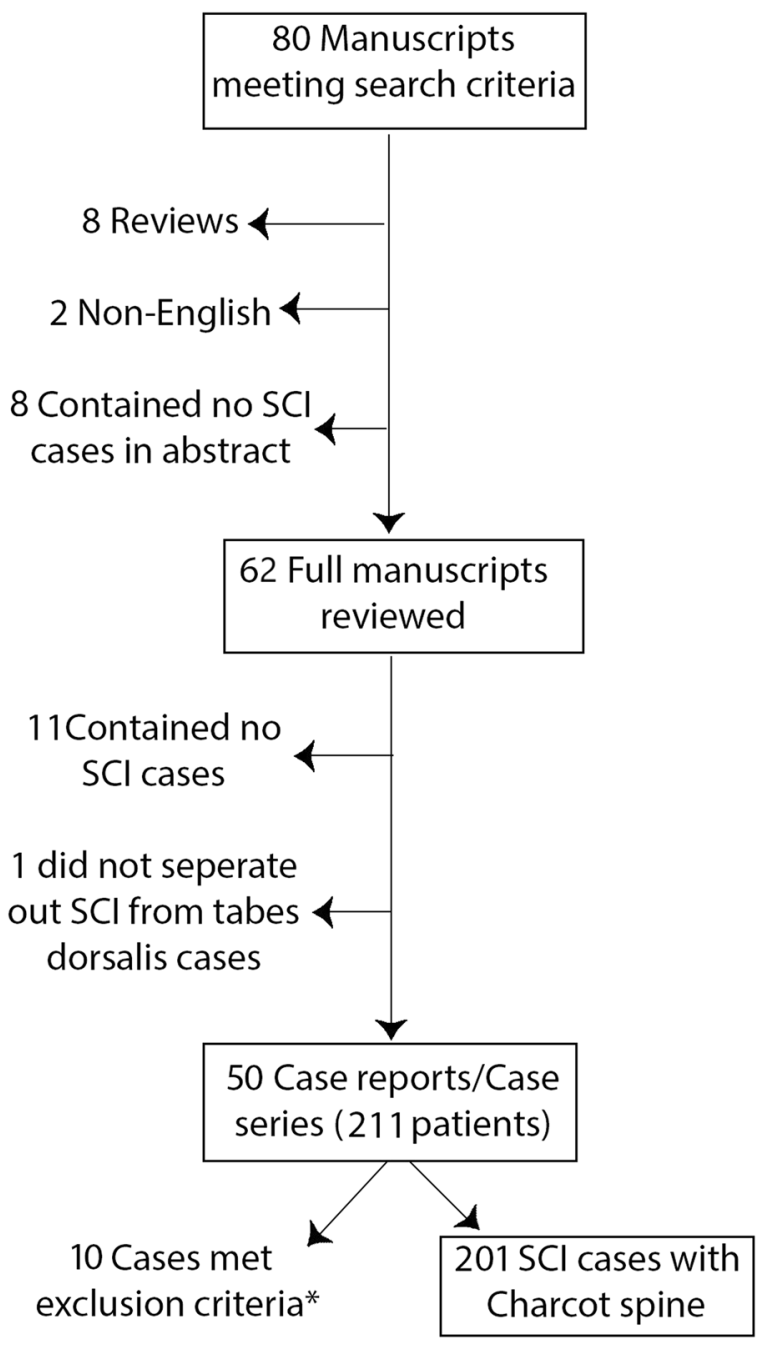

Fig. 1 Flow diagram of search for cases meeting inclusion criteria. *Excluded because of CIDP/GBS (4), Friedrich's ataxia (2), prior ankylosing spondylitis diagnosis (2), tabes dorsalis (1), fracture through laminectomy site (1)

results for 201 patients with preexisting SCI who developed Charcot spine were analyzed. Individual data were available for 189 of these cases, with one senior author being unable to be contacted (and thus data remaining aggregate). Full demographics for both these patients and the 12 reported as aggregate data appear in Table 1 . Notably, due to nonuniformity in data reporting in the initial papers, available data fluctuated in sample size and both individual and aggregate data were used when available. Mean length of initial spinal fusion spanned 7.7 vertebral bodies $(\mathrm{SD}=3.9$, $n=89$ ). Hip flexion restrictions were noted inconsistently within reports, and as such, was not included as a potential risk factor. Three injuries (1\%) were described only as "incomplete" and thus excluded from AIS specific analyses.

Patients presented with a variety of symptoms (Table 2), reports of which were included in $83 \%$ of all
Table 1 Demographics. AIS

\begin{tabular}{|c|c|c|}
\hline \multirow[t]{2}{*}{ Gender $(n=175)$} & Female & $24 \%(42)$ \\
\hline & Male & $76 \%(133)$ \\
\hline Age $(n=157)$ & $\begin{array}{l}\text { Mean } 41.4 \text { min, } \max \\
(12,74)\end{array}$ & \\
\hline \multirow[t]{5}{*}{ AIS $(n=145)$} & A & $93 \%(135)$ \\
\hline & B & $6 \%(8)$ \\
\hline & $\mathrm{C}$ & $1 \%(1)$ \\
\hline & $\mathrm{D}$ & $0 \%(0)$ \\
\hline & $\mathrm{E}$ & $1 \%(1)$ \\
\hline \multirow[t]{4}{*}{ NLI $(n=157)$} & Cervical & $14 \%(22)$ \\
\hline & T1-T6 & $30 \%(47)$ \\
\hline & $\mathrm{T} 7-\mathrm{T} 12$ & $53 \%(83)$ \\
\hline & L1-S4 & $3 \%(5)$ \\
\hline $\begin{array}{l}\text { Chronicity of injury at time of } \\
\text { CSA }(n=171)\end{array}$ & $\begin{array}{l}\text { Mean } 19.5 \text { years min, } \\
\max (1,43)\end{array}$ & $\mathrm{SD}=11.0$ \\
\hline $\begin{array}{l}\text { Delay from symptom onset to } \\
\text { diagnosis }(n=47)\end{array}$ & $\begin{array}{l}\text { Mean } 3.1 \text { years min, } \\
\max (0,13.3)\end{array}$ & $\mathrm{SD}=3.6$ \\
\hline
\end{tabular}

Due to heterogeneity of data reported, $n$ was variable for each category AIS American Spinal Injury Association (ASIA) Impairment Scale, NLI neurological level of injury, CSA Charcot spinal arthropathy

201 available cases. Notably, in cases of recurrence of Charcot spine, the presenting symptoms for the recurrent finding were rarely included. In patients classically at risk for autonomic dysreflexia (AD) based upon a NLI at or above T6, 13 of 73 patients (19\%) presented with $\mathrm{AD}$ when diagnosed with Charcot spine. Notably two patients with NLI of T9 and T10 also presented with reported AD. Of the $99 \%$ of cases where injury etiology was reported, $84 \%$ were documented in persons with an initial traumatic cause of their SCI, with the remaining cases due to nontraumatic etiology (ischemia, transverse myelitis, neoplasm, etc.). There were no differences in presenting symptoms of Charcot spine in patients with traumatic versus non-traumatic SCI $(p=0.009-0.352)$. Notably, spinal deformity did approach a statistically significant higher incidence in traumatic cases $(p=0.009)$, though did not meet requisite levels with Bonferroni correction ( $p<0.003$ needed).

Radiologic findings used to make the diagnosis of Charcot spine were provided in 92 cases and appear in Table 2. Patients with initial traumatic SCI were no more likely to have any specific radiographic finding on diagnosis of Charcot spine than the group with non-traumatic SCI. Crepitus, which has been hypothesized to be found more likely with pseudoarthrosis [4], was a presenting finding in $33 \%$ of cases with radiographic pseudoarthrosis. This contrasted with $32 \%$ of cases without radiographic evidence of pseudoarthrosis $(p=0.457)$. 
Table 2 Clinical and radiographic presentations of Charcot spine in patients with SCI

\begin{tabular}{llll}
\hline Clinical presentations $(n=166)$ & \multicolumn{3}{l}{ Radiologic findings $(n=92)$} \\
\hline Back pain & $56 \%(93)$ & Vertebral body destruction & $83 \%(76)$ \\
Spinal deformity & $48 \%(79)$ & Osteophytes & $61 \%(56)$ \\
Crepitus & $34 \%(57)$ & Endplate destruction & $57 \%(52)$ \\
Difficulty sitting & $30 \%(50)$ & Spinal instability & $45 \%(41)$ \\
Change in spasticity & $18 \%(30)$ & Paraspinal mass & $42 \%(39)$ \\
Neurological loss & $17 \%(29)$ & Vacuum phenomenon $^{\text {s }}$ & $17 \%(16)$ \\
Skin breakdown & $11 \%(18)$ & Other $^{\mathrm{b}}$ & $8 \%(7)$ \\
Autonomic dysreflexia & $10 \%(17)$ & & \\
Other & & & \\
Other pain & $6 \%(10)$ & & \\
\hline
\end{tabular}

$n=166$ for clinical presentations, $n=92$ for radiologic findings

${ }^{a}$ Other symptoms included fatigue (2), digestive discomfort (1), fever (1), frequent urinary tract infections (1), shortness of breath (1), orthostatic hypotension (1)

${ }^{\mathrm{b}}$ Other radiologic findings included kyphosis (3), scoliosis (3), stenosis (1), syrinx (1)

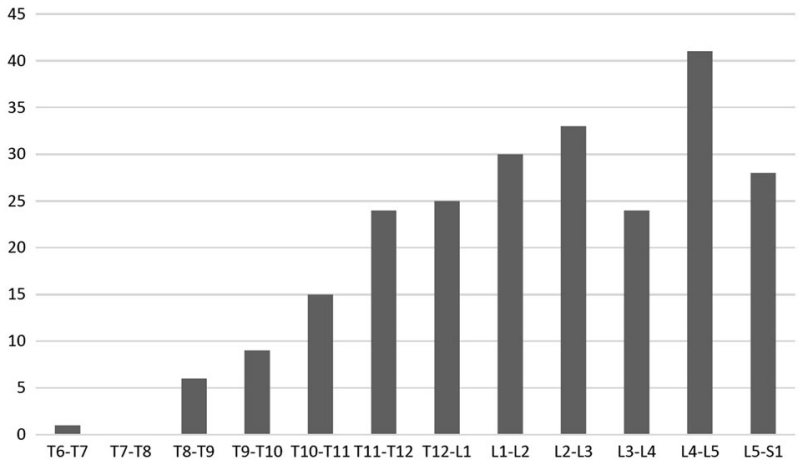

Fig. 2 Graph of incidence of levels involved in Charcot spine following SCI. $n=202$ reports where level of Charcot spine was available (including recurrence)

The most common level where Charcot spine had developed was L4-L5 (Fig. 2). In 33\% of cases, the involved joint was within the initial fusion and in $25 \%$ of cases, the involved joint was in the spinal joint immediately adjacent to the fusion. In 26 cases, Charcot spine involved multiple levels (26 at a second level, seven at a third level, and one at a fourth level). These additional Charcot spine segments were found in contiguous segments immediately below the most rostral Charcot spinal segment $73 \%$ of the time. In $51 \%$ of cases, there was explicit mention that a bone biopsy was taken to rule out osteomyelitis. Recurrence of Charcot spine, after initial diagnosis and management (either operative or nonoperative), occurred in 25 total cases $(19 \%)$. The mean time of follow up after intervention for monitoring for recurrence was 4.2 years.

Surgical management was pursued in $77 \%$ of cases, while the remainder $(23 \%, n=43)$ were non-operatively managed. Traumatic cases had initial fusion rate of $85 \%$ vs $65 \%$ in nontraumatic cases $(p=0.011)$. In cases where Charcot spine involved lumbar joints, fusion to at least the sacrum was performed in 44 of 95 cases. In the 51 cases where fusion was not done at least to the pelvis, secondary Charcot spine develop caudal to the fused segment in $14 \%$ of cases (versus $25 \%$ of cases where fusion was at least to the sacrum $p=0.079$ ). Non-operatively managed cases did not have any reported recurrence of Charcot spine during the mean of 3.6 years of clinical follow up.

\section{Discussion}

Based upon this analysis of reported cases, several important details are solidified regarding Charcot spine following SCI. As previously suggested, the vast majority (93\%) of reported Charcot spine cases occurred in individuals with neurologically complete SCI $[2,4,5,9]$. As more complete motor and sensory SCI is generally associated with a greater degree of autonomic dysfunction [10], altered autonomic innervation [11], and microvascular blood flow may further contribute to Charcot spine following SCI.

The majority of the reported cases of Charcot spine occurred in the lumbar region, with only $7 \%$ occurring above T10. This is in agreement with previous analyses, which note 5\% occurrence above this level in heterogenenous patient samples [7], and likely suggests shared mechanical stresses at these levels regardless of if Charcot spine is developed after a peripheral neuropathy or SCI. Despite the increasing number of patients surviving with tetraplegia following SCI, the majority of patients $(>86 \%)$ who were reported to develop Charcot spine after SCI had paraplegia. This argues that there may be something fundamental about patients with paraplegia (potentially the ability to perform independent, lateral transfers leading to excessive biomechanical loads or preferring a lower back on their wheelchairs) that predisposes this group to 
Charcot spine [5]. Further biomechanical research is required to identify these predisposing factors.

The most common symptom leading to diagnosis of Charcot spine was lower back pain. This finding of pain most commonly below a neurologically complete injury may seem counterintuitive, though deep pain can be due to directly spared local pain tracts (not all of which are assessed by the ISNCSCI exam) or experienced indirectly through symptoms of autonomic dysreflexia, As some studies indicate that lower back pain occurs in up to $61 \%$ of patients with SCI [12], this finding alone is likely insufficient to clinically suspect Charcot spine. Autonomic dysreflexia was also commonly reported (19\% in those at risk). Past reports have suggested that AD may result from "spinal instability exerting pressure on presacral plexus of nerves and retroperitoneal viscera" [13, 14]. However, a more likely explanation would be that $\mathrm{AD}$ is a response to local pain from the joint, even if not centrally perceived by the patient. $\mathrm{AD}$ as an identifying characteristic of Charcot spine is troubling, and given that the prevalence of asymptomatic "silent AD" is over 40\% [15], it is likely that the majority of patients at risk for $\mathrm{AD}$ who have Charcot spine experience some autonomically-induced hypertension.

Charcot spine, by radiologic definition should involve all three spinal columns [9, 16, 17] so the finding of spinal instability on imaging in $45 \%$ of cases is not surprising. Additionally, the presence of bridging osteophytes around the lesion and evidence of cartilaginous destruction ${ }^{3}$ was also commonly seen (61 and 56\% respectively). Others [18] have proposed that Charcot spine follows two phases; an initial atrophic form, marked primarily by destruction, that later progresses to a hypertrophic form with osteophyte formation. This fits other surgical literature that proposes progression from endplate destruction to vertebral body destruction and eventual pseudoarthrosis [4]. Prior research has further suggested that the presence of the pseudoarthrosis was the key finding that led to the clinical presentation of crepitus. Our findings did not support this link, as crepitus was similarly likely to be found on exam with or without radiographic evidence of pseudoarthrosis [4].

In patients who had an initial fusion for their SCI, Charcot spine developed in the directly adjacent segment to the fusion $25 \%$ of the time. Published cases in this analysis had a mean length of fusion of 7.7 spinal levels, well over the $>5$ level threshold previously outlined as a potential risk factor for adjacent segment disease to occur [4]. This skewed sample may limit conclusions made from these analyses and further research is needed to better determine the role of spinal fusions on development of Charcot spine.

Management of Charcot spine has varied over the years, with poor surgical results being one of the rare commonalities [19]. Recurrence of Charcot spine was found over 41 times more commonly than primary Charcot spine (19\% vs $0.5 \%)$.
While increased surveillance conceivably plays some role, additional predisposing factors are likely also at work. Many authors recommend both anterior and posterior fusion [4, 9]. In cases of lumbar Charcot spine, this involved fusion to the sacrum $(>46 \%)$. The higher rate of recurrence of Charcot spine in those lumbar cases where fusion was performed to the pelvis is of unclear significance, as many factors contributing to selection of operative management. This however, should at least raise the question of appropriateness of such recently advocated extended fusions [19, 20]. Given the significant changes in surgical technology over the half a century encompassed by this analysis, further extrapolations on efficacy of management are outside of the appropriate scope of this study.

\section{Limitations}

Cases were identified using Google Scholar in an effort to capture all potentially non-indexed studies. However, it is possible that these methods were insufficient and additional cases of Charcot spine after SCI exist. Pooled analyses, as were completed to characterize our data, may underestimate actual frequency of symptoms as standard assessments are not employed [21]. The variation in age of the studies and accompanying ISNCSCI classifications, imaging techniques, and surgical practices, presents an additional limitation in the findings. The multi-decade timeframe means that the ISNCSCI exam has undergone multiple revisions, including the altered definitions of complete injuries. However, given that the clear majority of these reported cases noted "complete" injuries, this is less likely to impact the findings.

\section{Conclusions}

Charcot spine may occur following SCI and commonly presents with lower back pain, spinal deformity, and crepitus. Radiographically, Charcot spine is often diagnosed with findings of vertebral body/endplate destruction and osteophytes. The recurrence rate of Charcot spine is high following management, potentially making this complication of spinal cord injury especially debilitating.

Acknowledgements We would like to especially thank Dr. Jörg Krebs for his help in providing deidentified individual data from pertinent aggregate Charcot spine case series.

Author contributions RS was responsible for designing the review protocol, data extraction and analysis, interpreting results, and manuscript preparation. JMD was responsible for designing the review protocol, data verification, interpreting results, and manuscript preparation. SCK was responsible for designing the review protocol, interpreting results, and manuscript preparation. 


\section{Compliance with ethical standards}

Conflict of interest The authors declare that they have no conflict of interest.

\section{References}

1. Johnson JTH. Neuropatbic fractures and joint injuries: pathogenesis and rationale of prevention and treatment. J Bone Joint Surg. 1967;49-A:1-30.

2. Standaert CJ, Cardenas DD, Anderson PA. Charcot spine as a late complication of traumatic spinal cord injury. Arch Phys Med Rehabil. 1997;78:221-5.

3. Zyck S, Toshkezi G, Pizzuti J, Marawar S. Four-rod instrumentation for treatment of charcot spinal arthropathy causing autonomic dysreflexia: case report and literature review. Cureus. 2016;8:e850.

4. Vaccaro AR, Silber JS. Post-traumatic spinal deformity. Spine. 2001;26(24S):S111-8.

5. Aebli N, Pötzel T, Krebs J. Characteristics and surgical management of neuropathic (Charcot) spinal arthropathy after spinal cord injury. Spine J. 2013;14:884-91.

6. Krebs J, Grasmück D, Pötzel T, Pannek J. Charcot arthropathy of the spine in spinal cord injured individuals with sacral deafferentation and anterior root stimulator implantation. Nuerourol Urodyn. 2014;35:241-5.

7. Barrey C, Massourides H, Cotton F, Perrin G, Rode G. Charcot spine: two new case reports and a systematic review of 109 clinical cases from the literature. Ann Phys Rehabil Med. 2010;53:200-20.

8. Kirshblum S, Waring W. Updates for the international standards for neurological classification of spinal cord injury. Phys Med Rehabil Clin N Am. 2014;25:505-17.

9. Aydinli U, Mohan N, Kara K. Post-traumatic charcot (neuropathic) spinal arthropathy at cervicothoracic junction: case report. World Neurosurg. 2016;94:580e1-4.
10. Garstang SV, Miller-Smith SA. Autonomic nervous system dysfunction after spinal cord injury. Phys Med Rehabil Clin N Am. 2007;18:275-96.

11. Dudley-Javoroski S, Shields RK. Muscle and bone plasticity after spinal cord injury: Review of adaptations to disuse and to electrical stimulation. J Rehabil Res Dev. 2008;45:283-96.

12. Turner JA, Cardenas DD, Warms CA, McClellan CB. Chronic pain associated with spinal cord injuries: a community survey. Arch Phys Med Rehabil. 2001;82:501-8.

13. Thumbikat P, Ravichandran G, McClelland MR. Neuropathic lumbar spondylolisthesis - a rare trigger for posture induced autonomic dysreflexia. Spinal Cord. 2001;39:564-7.

14. Morita M, Iwasaki M, Okuda S, Oda T, Miyauchi A. Autonomic dysreflexia associated with Charcot spine following spinal cord injury: a case report and literature review. Eur Spine J. 2010;19 (sup2):S179-82.

15. Linsenmeyer TA, Campagnolo DI, Chou IH. Silent autonomic dysreflexia during voiding in men with spinal cord injuries. J Urol. 1996;155:519-22.

16. Denis F. The three column spine and its significance in the classification of acute thoracolumbar spinal injuries. Spine. 1983;8:817-31.

17. Jones EA, Manaster BJ, May DA, Disler DG. Neuropathic osteoarthropathy: diagnostic dilemmas and differential diagnosis. Radiographics. 2000;20:S279-93.

18. Brown CW, Jones B, Donaldson DH, Akmakjian J, Brugman J. Neuropathic (Charcot) arthropathy of the spine after traumatic spinal paraplegia. Spine. 1992;17(6S):S103-8.

19. Haus BM, Hsu AR, Yim ES, Meter JJ, Rinsky LA. Long-term follow up of the surgical management of neuropathic arthropathy of the spine. Spine J. 2010;10:e6-16.

20. Kasten MD, Rao LA, Priest B. Long-term results of iliac wing fixation below extensive fusions in ambulatory adult patients with spinal disorders. J Spinal Disord Tech. 2010;23:e37-42.

21. Lyman GH, Kuderer NM. The strengths and limitations of metaanalyses based on aggregate data. BMC Med Res Methodol. 2005;5:14. 